\title{
5 Zusammenfassung und Diskussion
}

In dieser Arbeit wurden zwei Arten von graphematischen Regularitäten untersucht, graphemische und morpho-graphematische. Im Folgenden werden wesentliche Ergebnisse dieser Untersuchung noch einmal zusammengetragen und diskutiert. Dabei soll zunächst dem Reihentitel Rechnung getragen werden: Wo konvergieren das deutsche und das englische Schriftsystem, wo divergieren sie? Was folgt, ist keine vollständige Übersicht über alle untersuchten Phänomene, sondern eine Auswahl besonders eindrücklicher Beispiele.

Überraschend ähnlich sind sich die beiden Systeme in der relativen Frequenz der Buchstaben (vgl. Abb. 1 oben, hier wiederholt als Abb. 49):

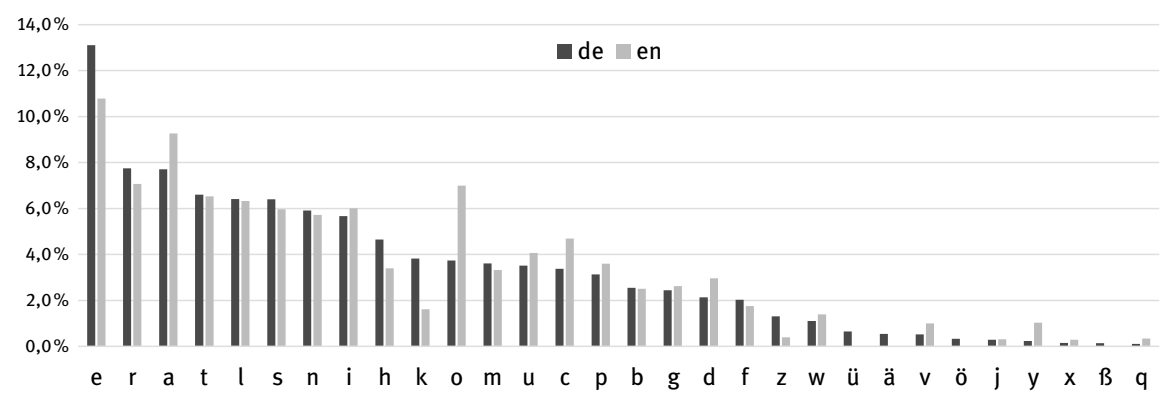

Abb. 49: Relative Anteile der Buchstaben im deutschen und englischen Teilkorpus einfacher Stämme an der Summe aller Buchstaben im jeweiligen Korpus. N(de): 30.772, N(en): 37.519.

Die relative Häufigkeit einiger Buchstaben weicht voneinander ab; so sind $|\mathrm{e}|,|\mathrm{h}|$, $|k|$ und $|z|$ im Deutschen etwas häufiger und $|a|,|c|,|o|$ und $|y|$ im Englischen. Insgesamt ist der Grad der Übereinstimmung aber bemerkenswert. Das schlägt sich in einem hohen Grad der Korrelation nieder (Pearsons $r=0,93, p<0,001$ ).

Auch die belegten und nicht belegten Bigramme (die Verbindungen von zwei Buchstaben) weisen große Übereinstimmungen auf. 90\% aller Bigramme, die im deutschen Korpus belegt sind, finden sich auch im englischen Korpus (siehe 3.2.1.3). Eine besonders interessante Teilklasse der Bigramme sind Verdoppelungen. Diejenigen Verdoppelungen, die signifikant häufiger sind als erwartet, stimmen größtenteils überein (Abb. 50). 


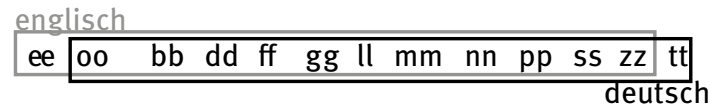

Abb. 50: Verdoppelungen von Buchstaben, die signifikant häufiger sind, als stochastisch zu erwarten wäre.

Die Verdoppelung von Konsonantenbuchstaben ist in beiden Systemen zentral, und die Schnittmenge ist groß. $|\mathrm{tt}|$ ist im Englischen nicht häufiger als erwartbar - aber auch nicht seltener. Bei den Vokalen ist der Unterschied auf den ersten Blick ebenfalls klein. Im Deutschen ist nur |ool häufiger als erwartet, im Englischen darüber hinaus auch |ee|. Diese Beschreibung verwischt aber die Tatsache, dass |oo| im Deutschen nur etwas häufiger ist, als zu erwarten wäre, während |ee| und |oo| im Englischen wesentlich häufiger sind. Die Verdoppelung von Vokalbuchstaben ist im Englischen zentraler als im Deutschen.

Typische Strukturen von Stämmen und Affixen hingegen konvergieren in den beiden Systemen weitestgehend. Die folgende Liste von Konvergenzen ist nicht abschließend:

- Die meisten Stämme in den Korpora sind zweisilbig, die meisten Affixe einsilbig.

- Die meisten einsilbigen Stämme haben einen einfach besetzten Anfangsrand, einen einfach besetzten Kern und einen zweifach besetzten Endrand ( $(\mathrm{CVCC})$ ).

- Jede Abweichung vom Prototyp (mehr oder weniger besetzter Anfangsrand, Kern, Endrand) führt zu einer Abnahme der Belegzahlen: Es gibt bspw. mehr einsilbige Wörter vom Typ 〈CVCC〉 als vom Typ 〈CCVCC〉, und von diesem Typ wiederum mehr als vom Typ «CCVC etc.

- Die meisten einsilbigen Affixe haben einen leeren Anfangsrand und einen einfach besetzten Kern und Endrand ( $(\mathrm{VC})$ ).

- Es gibt nur wenige Überschneidungen zwischen einsilbigen Stämmen und Affixen. Anders ausgedrückt: Die meisten Affixe sehen wie Affixe aus, die meisten Stämme wie Stämme. Die Struktur 〈CVC〉 (z. B. 〈Bus〉, 〈-nis〉, 〈gas〉, «-ful») ist die einzige Struktur, in der Stämme und Affixe quantitativ vorkommen (Abb. 46 oben, hier wiederholt als Abb. 51). 


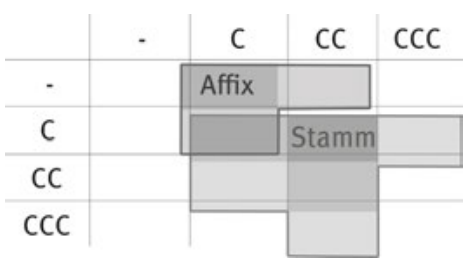

Abb. 51: Die Verteilung prototypischer einsilbiger Stämme und einsilbiger Affixe im Deutschen und Englischen.

Eine deutsche Besonderheit ist die Interaktion zwischen Anfangs- und Endrand. Bestimmte Grapheme kommen (in Autosemantika) nicht alleine im Endrand vor, wenn der Anfangsrand nicht oder nur einfach besetzt ist ( ${ }^{\star}$ Or $\rangle$, * ${ }^{\star}$ fül[en]〉). In diesem Kontext tritt das sog. Dehnungs-〈h〉 auf; es reguliert das Silbengewicht, indem es den Endrand schwerer macht (〈Ohr〉, 〈fühl[en]〉). Genauso kann auch das silbeninitiale $\langle\mathrm{h}$ 〉 interpretiert werden: Es beschwert Stämme, die ansonsten die Minimalitätsbedingung des zweifach besetzten Reims nicht erfüllen würden (z. B. 〈Schuh〉, * $\langle$ Schu〉). Interessant ist in diesem Zusammenhang, dass die Domäne der $\langle\mathrm{h}\rangle$-Schreibungen nicht das Wort oder der Stamm ist, sondern die Wurzel - also die Form, die nach Abzug der Endung übrigbleibt. So kann auch die 〈h〉-Schreibung in Fällen wie «Sahne〉 erfasst werden: Der Endrand der Wurzel ${ }^{\star} «$ San-〉 ist zu leicht, deswegen wird sie mit «h〉 beschwert («Sahn-〉).

Eine solche Segmentierung in Wurzel und Endung (die im Deutschen häufig ein Pseudosuffix ist, also 〈e〉, 〈er〉, 〈en〉 und 〈el〉) drängt sich in beiden Systemen auf. Neben der Tatsache, dass sich die Beschränkungen über das Gewicht der Silbenkonstituenten nicht auf Stämme, sondern auf Wurzeln beziehen, spricht für eine solche Gliederung auch, dass Wurzeln häufig dieselbe Struktur haben wie einsilbige Stämme - 〈Sahn-〉 ist aufgebaut wie «Hahn〉. Das gilt allgemein für die typischen CV-Strukturen, es gilt aber auch konkret für die finalen Konsonantencluster in Wurzeln und Stämmen. Darüber hinaus konnte gezeigt werden, dass die Endungen (die ja definitorisch Reime sind) häufig weniger kompositionell sind als Reime von Einsilbern.

Die minimalen Strukturen im Deutschen und Englischen stimmen ebenfalls weitestgehend überein:

- Stämme (etwas genauer: autosemantische Stämme) bestehen aus mindestens drei Graphemen, und ihr Reim ist mit mindestens zwei Graphemen besetzt (〈VCC〉, 〈CVC〉 oder 〈CVV〉).

- Sprachspezifisch sind in diesem Zusammenhang nur wenige Beschränkungen: Im Englischen verhält sich 〈y〉 wie zwei Grapheme (der Typ 〈dry〉 braucht kein weiteres Graphem im Kern oder Endrand). Im Deutschen sind einfaches $\langle\mathrm{l}\rangle,\langle\mathrm{m}\rangle,\langle\mathrm{n}\rangle$ und $\langle\mathrm{r}\rangle \mathrm{zu}$ leicht; im Englischen sind es $\langle\mathrm{f}\rangle,\langle\mathrm{l}\rangle$ und $\langle\mathrm{s}\rangle$. 
- Affixe und Synsemantika sind leichter: Präfixe bestehen aus mindestens zwei Graphemen (〈VC〉 oder 〈CV〉); bei Suffixen gilt zusätzlich, dass der Reim zweifach besetzt sein muss ( $\langle\mathrm{VC}\rangle)$.

- Synsemantika unterscheiden sich im Englischen und Deutschen: Im Englischen gilt lediglich, dass der Kern einfach besetzt sein muss ( \langle\rangle$)$, im Deutschen darüber hinaus, dass die Struktur aus zwei Graphemen besteht (〈VC〉 oder $\langle\mathrm{CV}\rangle)$.

Das sind sehr weitgehende Konvergenzen, wenn man bedenkt, wie unterschiedlich konsistent die Schriftsysteme auf der segmentalen Ebene sind. Das deutsche Schriftsystem ist wesentlich näher an eineindeutigen Phonem-Graphem-Korrespondenzen als das englische, das ja gemeinhin als chaotisch oder - etwas neutraler - als stark idiosynkratisch gilt. Dass vor allem auf der suprasegmentalen Ebene so große Übereinstimmungen bestehen, ist überraschend.

Divergenzen finden sich vor allem, was die morphographische Schnittstelle anbelangt. Hier wurden mit der Einheitlichkeit und der Eindeutigkeit zwei Parameter angesetzt, die im weiteren Sinn auf das Form-Funktions-Verhältnis abheben. Ein Morphem ist einheitlich, wenn es nur eine graphematische Form gibt, die das Morphem repräsentiert. Und eine solche graphematische Form ist wiederum eindeutig, wenn jede Instanz dieser Form auf dasselbe Morphem verweist. Diese Parameter wurden für Stämme und Affixe im Englischen und Deutschen überprüft.

Es zeigt sich, dass im Deutschen die Einheitlichkeit von Stämmen wichtig ist, und zwar a) wichtiger als die Einheitlichkeit von Affixen und die Eindeutigkeit von Stämmen und Affixen im Deutschen und b) wichtiger als die Einheitlichkeit von Stämmen im Englischen. Die Einheitlichkeit von Stämmen ist im Deutschen ein entscheidendes Strukturprinzip. Das könnte einen Verarbeitungsvorteil haben - Stämme sind leichter wiedererkennbar, wenn sie formal nicht variieren. Ackermann/Zimmer (2017) zeigen empirisch, dass das zumindest für bestimmte Substantive im Deutschen gilt.

Im Englischen ist umgekehrt die Eindeutigkeit von Affixen entscheidend. Auch für diese morphographische Schreibung kann ein Verarbeitungsvorteil angenommen werden: Wenn eine bestimmte Schreibung immer ein Affix repräsentiert und nie ein zufälliges Wortende, dann liefert die Schrift den Lesern morphologische und morphosyntaktische Informationen auf dem Silbertablett: Die Leser sehen auf den ersten Blick, dass es sich bei einem bestimmten Teil eines graphematischen Wortes um grammatische und nicht um lexikalische Informationen handelt. In den meisten Fällen handelt es sich hier um Distinktivität: Eine Schreibung repräsentiert exklusiv morphologische Information und genau kein Wortende; um welches Affix es sich konkret handelt, ist aber oft nicht spezifi- 
ziert. Eindeutigkeit im engeren Sinne, also der Verweis auf ein und nur ein Affix, ist die Ausnahme.

Auch für die Einheitlichkeit von Affixen gibt es im Englischen wesentlich weniger Ausnahmen als im Deutschen - und vor allem wird eine Reihe von morpho-phonologischen Alternationen graphematisch überbrückt (man denke nur an die Flexionssuffixe -s und -ed oder das Derivationssuffix -ic, die alle graphematisch einheitlicher sind als phonologisch).

Die Eindeutigkeit von Stämmen ist in beiden Sprachen zwar weit verbreitet; sie kann als der Normalfall gelten. Es gibt aber in beiden Sprachen einen nichtignorierbaren Teil an Ausnahmen - 3,3\% homographischer Formen im Deutschen, 4,9\% im Englischen. Wichtiger ist: Es werden im Englischen nur etwa die Hälfte aller homophonen Formen graphematisch differenziert (z. B. meet/meat); die andere Hälfte ist homographisch (z. B. date ${ }_{1} /$ date $_{2}$ ). Im Deutschen liegt dieser Anteil noch einmal niedriger, und zwar bei weniger als einem Fünftel aller Homophone. Es wird also längst nicht überall graphematisch differenziert, wo das lexikalisch möglich wäre.

Insgesamt zeigt sich, dass im Englischen eher Affixe verlässlich kodiert werden (sie sind oft eindeutig und einheitlich), während es im Deutschen Stämme sind, die häufig einheitlich kodiert werden. Das sind zwei grundsätzlich unterschiedliche Strategien der Leseerleichterung. Im Deutschen wird der lexikalische Zugriff potenziell erleichtert, im Englischen der Aufbau grammatischer Strukturen (indem bspw. anhand eines Derivationssuffixes die Wortart ablesbar ist).

Welche Kriterien und Methoden eignen sich besonders für einen Vergleich von Schriftsystemen? Diese Frage wurde eingangs gestellt (siehe 1.1); sie soll hier kurz diskutiert werden. Die Voraussetzung ist sicherlich, dass sich für die Sprachen, die untersucht werden sollen, eine elektronische Liste der Stämme aufstellen lässt. Das ist die Minimalanforderung. Eine elektronische Datenbank, die auch phonologische und morphologische Informationen enthält, lässt weitere Untersuchungen zu; aber alleine mit einer Liste einfacher Stämme kann bereits eine Vielzahl von Informationen erhoben werden.

Das betrifft zum einen die quantitative Verteilung der Buchstaben. Welche Buchstaben sind häufig, welche selten? Das Deutsche und das Englische stimmen weitgehend überein, was die relativen Anteile der Buchstaben angeht (3.1); es wäre interessant zu wissen, ob das in anderen Sprachen ebenso ist und worauf sich die Gemeinsamkeiten oder Unterschiede zurückführen lassen. Es handelt sich hier um einen sehr groben Parameter - welche Buchstaben sind unter allen einfachen Stämmen wie häufig? - und dennoch lassen sich eventuell interessante Rückschlüsse ziehen.

Zweitens können die Buchstaben automatisch in Konsonanten und Vokale eingeteilt werden, und zwar automatisch auf der Grundlage ihrer syntagmati- 
schen Verteilung. Dieses Verfahren ist etwas ausgefeilter als die reine quantitative Verteilung. Mit ihm lassen sich Aussagen über die Inventare von Vokalen und Konsonanten in der betreffenden Sprache machen, und diese Inventare können wiederum mit den etablierten (und aller Wahrscheinlichkeit nach phonographisch motivierten) Inventaren verglichen werden.

Drittens kann die Verteilung der Bigramme untersucht werden; auch dafür ist lediglich eine Liste mit der schriftlichen Form der Stämme notwendig. Wir können dann für jedes Bigramm feststellen, ob es wesentlich häufiger oder seltener auftritt, als das stochastisch zu erwarten wäre. Ein Sonderfall (allerdings ein sehr salienter) sind Verdoppelungen von Buchstaben: Welche Buchstaben können verdoppelt werden, welche nicht? Außerdem können mögliche und unmögliche Stammenden ermittelt werden.

Viertens kann die Liste der Stämme mit silbenstrukturellen Informationen angereichert werden, die wiederum auf der Einteilung in Vokale und Konsonanten beruhen. Mit diesen Informationen lassen sich nun sehr einfach Aussagen über die Anzahl der Schreibsilben sowie häufige und seltene CV-Strukturen machen. Außerdem kann die Besetzung der Silbenkonstituenten graphotaktisch beschrieben werden, und die Konstituenten können auf Interaktionseffekte untersucht werden.

Und fünftens kann schließlich überprüft werden, wie minimale Strukturen aussehen (gibt es eine Mindestlänge oder andere Mindestanforderungen für alle Stämme? Für die autosemantischen Stämme?) und wie Stämme typischerweise aussehen.

Für die Untersuchung der Einheitlichkeit und Eindeutigkeit sind dann morphologische und phonologische Informationen (und vor allem ein gewisses morphologisches Hintergrundwissen der jeweiligen Sprache) erforderlich. Dann kann aber für Stämme und Affixe festgestellt werden, wie einheitlich sie sind, und für jede graphematische Form, wie eindeutig bzw. distinktiv sie sind. Hier ist jeweils ein Vergleich mit der Phonologie instruktiv: Wo werden morpho-phonologische Alternationen überbrückt, wo nicht? Wo sind distinkte Lexeme homophon, aber heterographisch? Wo nicht?

So unterschiedlich die Untersuchungen sind, die in dieser Arbeit durchgeführt wurden - sie haben doch eines gemein: Sie beschreiben alle den synchronen Zustand des Schriftsystems. So kann expliziert werden, wie das System heute funktioniert; es bleibt aber unklar, wie die heutige Form des Systems sich herausgebildet hat.

Im Folgenden soll schlaglichtartig gezeigt werden, wie eine quantitative Untersuchung der diachronen Entwicklung aussehen könnte und was sie zu leisten vermag. Ausgangspunkt ist die Teiluntersuchung in Berg/Aronoff (2017), die sich mit der Herausbildung der Suffixschreibungen beschäftigt, und zwar für 
dieselben vier Derivationssuffixe, deren Verteilung synchron ermittelt wurde (-ous, -ic, -al und -y). Die Datengrundlage bildete das relativ kleine diachrone Helsinki-Korpus (vgl. Helsinki Corpus of English Texts 1991), das nicht lemmatisiert ist und grobe Zeitangaben in 70-Jahres-Schnitten (bis 1710) enthält. Für jedes Suffix wurde mithilfe des OED die Menge der Schreibvarianten ermittelt (für -ic bspw. 〈ic〉, 〈ik〉, 〈ike〉, 〈ique〉, 〈icke〉, 〈ick〉 etc.). Im Helsinki-Korpus wurden dann alle Wörter (i. S. v. Token, also laufende Textwörter) gesucht, die mit diesen Graphemfolgen enden (z. B. 〈panick〉, 〈catholicke〉), und für jedes dieser Wörter wurde überprüft, ob es heute mit 〈ic〉 endet. Daraus ergibt sich dann für jeden Zeitschnitt ein ,Spektrum' der Schreibungen (z. B. 1350-1420: 65 \% 〈ik〉, $20 \%$ 〈ic〉, $10 \%$ <ike〉 etc.).

In Abbildung 52 sind die relativen Anteile der Schreibvarianten über die Zeit aufgetragen (nach Berg/Aronoff 2017; für die Zeit ab 1710 wurde das wesentlich größere Korpus von Google Books verwendet, vgl. https://books.google.com/ ngrams, Stand: 22.11. 2018):

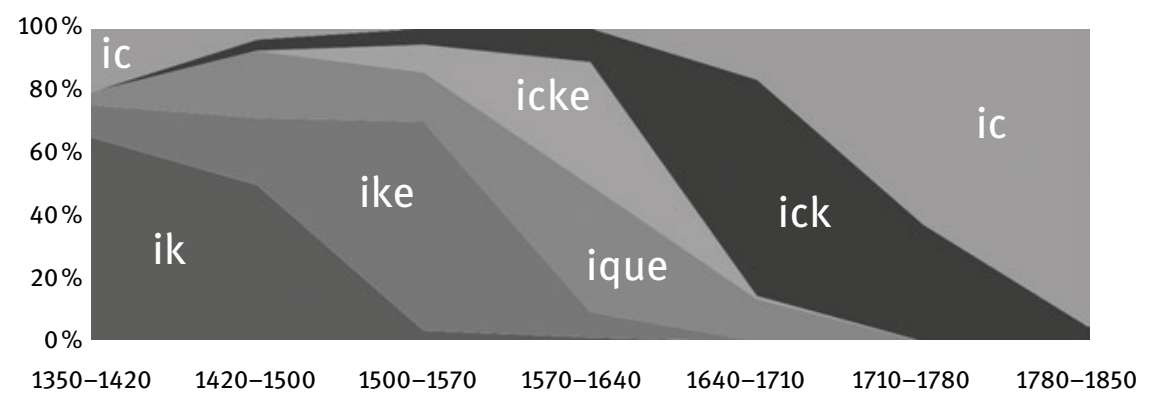

Abb. 52: Relativer Anteil der Schreibvarianten des Suffixes -ic bei Wörtern, die heute mit 〈ic〉 geschrieben werden. Datenbasis: Helsinki-Korpus (1350-1710) und Google Ngrams (1710-1850).

In beinahe jedem Zeitabschnitt ist eine andere Form dominant («ik〉>〈ike〉 \rangle$\langle$ icke $\rangle\rangle\langle$ ick $\rangle\rangle\langle$ ic $\rangle$ ). Die heutige Schreibung $\langle$ ic $\rangle$ ist zwar im ersten Zeitschnitt bereits vertreten, verschwindet dann aber für ein paar Jahrhunderte und setzt sich erst 1710-1780 durch. Anders als bei anderen Suffixschreibungen, z. B. -ous, wird hier Variation nicht kontinuierlich abgebaut - es treten im Gegenteil zeitweise neue Varianten auf (iicke〉 z. B. erstmals 1500-1570).

Das Helsinki-Korpus ist relativ klein; es umfasst knapp 1,5 Millionen Token. Daher können viele interessante Fragen der Verteilung nicht mit diesem Korpus bearbeitet werden: Wie genau läuft der Übergang von einer zur anderen Variante ab? Ist er diatopisch zu motivieren, wird also eine regionale Variante in den Stan- 
dard übernommen? Oder lassen sich ,Leuchttürme“ ausmachen - Schreiber, die eine bestimmte Variante verhältnismäßig früh verwenden und von denen wir wissen, dass sie einflussreich waren? Die Auflösung des Helsinki-Korpus ist einfach zu klein, um diese Fragen zu beantworten.

Hier bieten sich größere diachrone Korpora wie das Korpus ,Early English Books Online“ (EEBO, 1475-1700) und das Korpus ,Eighteenth Century Collections Online، (ECCO, 1700-1799) an (www.textcreationpartnership.org, Stand: 22.11. 2018); für das Deutsche ist das Deutsche Textarchiv einschlägig (www. deutschestextarchiv.de, Stand: 22.11.2018). Das EEBO-Korpus umfasst knapp 500 Millionen Token, das ECCO-Korpus 75 Millionen Token. Die zeitliche Auflösung ist viel höher, und zusätzlich liegen für jeden Text Informationen zum Autor, Verlag und Ort (sowie z. T. Drucker) vor, die für datengeleitete Analysen genutzt werden können.

Führt man die Untersuchung aus Berg/Aronoff (2017) in den EEBO- und ECCO-Korpora durch, so ergibt sich ein sehr ähnliches Bild wie im HelsinkiKorpus:

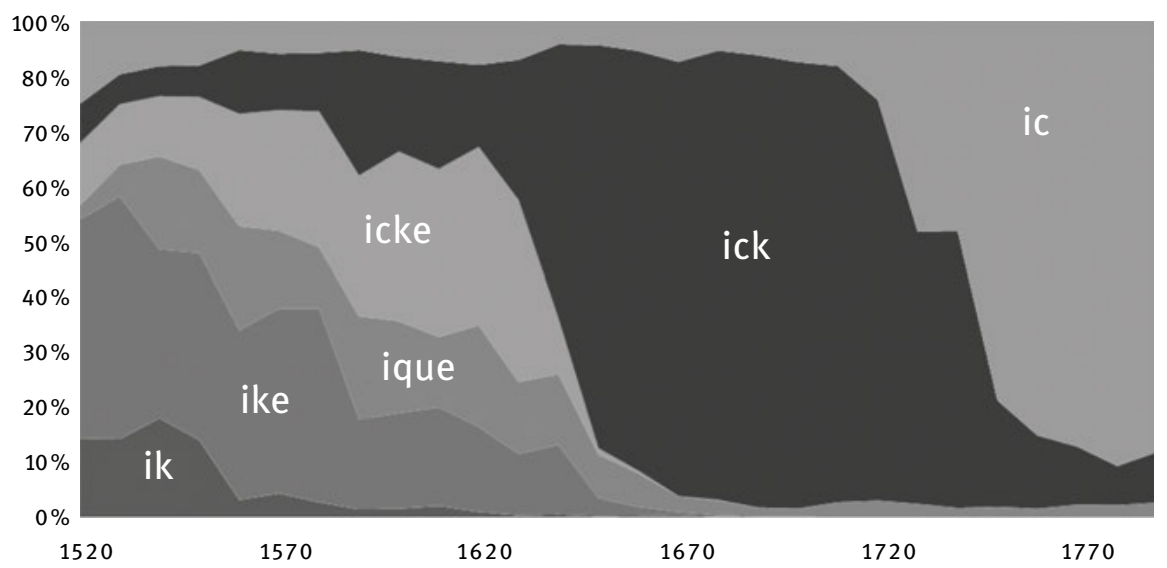

Abb. 53: Relativer Anteil der Schreibvarianten des Suffixes -ic bei Wörtern, die heute mit «ic geschrieben werden. Datenbasis: EEBO-Korpus (bis 1700) und ECCO-Korpus (ab 1700).

Durch die höhere zeitliche Auflösung sind die Übergänge weniger glatt. Anders formuliert: Es können auch kleinere Schwankungen abgebildet werden. Ansonsten stimmen die Abbildung 52 und 53 überein, was die ungefähren Anteile der jeweiligen Varianten sowie die zeitliche Abfolge angeht. Das ist einerseits eine Bestätigung der Ergebnisse aus Berg/Aronoff (2017); andererseits ist es eine Ermutigung, auch mit kleineren diachronen Korpora zu arbeiten. 
Wir können hier aber nun weitergehen und die Daten etwas genauer betrachten. Das geschieht in Abbildung 54, die den Übergang von 〈ick〉 $\mathrm{zu}$ 〈ic〉 zwischen 1650 und 1800 darstellt. Jeder Punkt im Streudiagramm ist ein Text, und die vertikale Achse zeigt an, wie hoch der Anteil der 〈ick〉-Formen im jeweiligen Text ist.

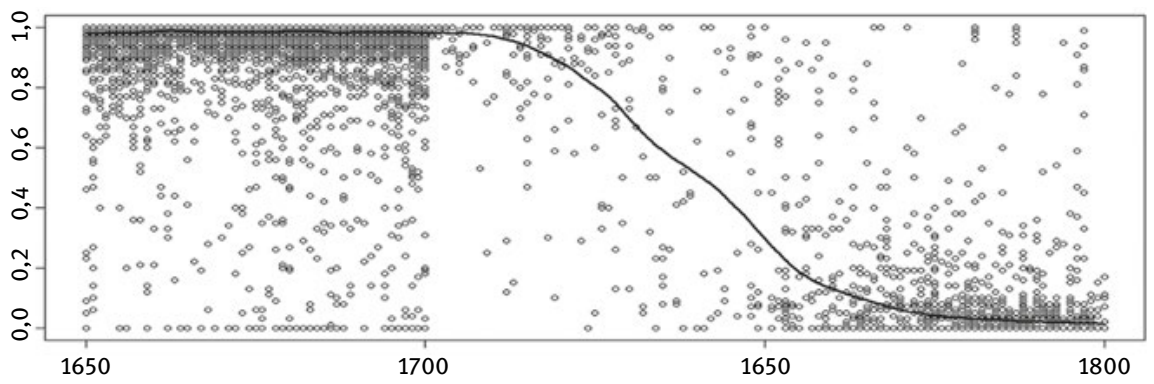

Abb. 54: Streudiagramm von Texten aus dem EEBO- und dem ECCO-Korpus. Horizontale Achse: Jahr der Veröffentlichung. Vertikale Achse: Relativer Anteil der «ick〉-Formen im jeweiligen Text. Linie: Mittelwert der relativen Anteile pro Jahr.

Am Anfang ist 〈ick〉 absolut dominant, dann gibt es eine etwa 70-jährige Übergangsperiode, an dessen Ende 〈ic〉 dominiert (die Linie zeigt die Mittelwerte des Anteils von 〈ick a an allen Token eines Jahres an). Der gut sichtbare Bruch um 1700 ist die Grenze der beiden Korpora: Das EEBO-Korpus ist sehr viel dichter als das ECCO-Korpus, das 1700 beginnt. Auch wenn es auf den ersten Blick so aussieht, als ob die Variation schlagartig nachlässt - das ist wohl nur ein Effekt der geringeren Textdichte pro Jahr nach 1700. Wenn es mehr Text gäbe, gäbe es auch mehr Variation.

Die einzelnen Texte können nun danach klassifiziert werden, wo sie gedruckt und/oder veröffentlicht wurden oder wer sie geschrieben hat. Wir können außerdem von der Verteilung in Abbildung 54 ausgehen und die Autoren der Texte in graphematische Innovatoren und Traditionalisten einteilen. Innovatoren sind im konkreten Fall solche Schreiber, die 〈ic〉 verwenden, noch bevor die Mehrheit das tut (also vor etwa 1740). Traditionalisten sind andersherum solche Schreiber, die ¿ick〉 noch verwenden, nachdem die Mehrheit das nicht mehr tut (also nach ca. 1740). Und tatsächlich finden sich unter den Innovatoren viele einflussreiche Schreiber (z. B. der Theologe George Berkeley oder der Schriftsteller Alexander Pope, die zwischen 1710 und 1740 Texte veröffentlicht haben, in denen die neue Schreibung überwiegt). Umgekehrt stammen viele der Texte in ,traditioneller Orthographie im rechten oberen Bereich des Diagramms vom Schriftsteller Edmund Burke, einem der Vordenker des Konservativismus. Burke verwendet um 
1800 noch eine Schreibung, die die meisten anderen Schreiber bereits knapp 60 Jahre vorher aufgegeben haben. Diese und andere Untersuchungen stehen noch am Anfang; es ist aber zu hoffen, dass mit einem umfangreichen diachronen Korpus und den richtigen Analysemethoden neue Erkenntnisse zum Ablauf graphematischer Wandelprozesse gewonnen werden können. 